\title{
What Can We Save for the First-Line Treatment of NSCLC in $2016 ?$
}

\author{
David Rossi
}

Lung cancer is the leading cause of cancer-related deaths worldwide. In the United States, there were 221,200 new diagnoses and 158,400 deaths in 2015, according to the National Cancer Institute. In these last years, histologic definition has become crucial in the therapeutic algorithm of NSCLC. Today we recognize three big groups of lung cancer: non-squamous "wild type", non-squamous with genetic mutations and squamous. For the non-squamous "wild type", pemetrexed plus cisplatin or carboplatin is the gold standard of care with chance of pemetrexed maintenance in patients with responsive or stable disease; another option is the addition of bevacizumab to a doublet with paclitaxel/carboplatin. For the non-squamous with genetic mutations, we can take into account clinical practice of epidermal growth factor receptor (EGFR) mutations, ALK translocations and ROS-1 rearrangements: gefitinib, erlotinib and afatinib for EGFR mutations, crizotinib for ALK translocations and ROS-1 rearrangements. For squamous cell carcinoma, a doublet with platinum derivate plus gemcitabine, vinorelbine or docetaxel is the gold standard; last year, necitumumab (a new anti-EGFR monoclonal antibody) has been approved by FDA in combination with cisplatin and gemcitabine (SQUIRE trial). In these last 2 years, immunocheck-point inhibitors documented high activity as second line in squamous and non-squamous cell carcinoma. In 2015, FDA approved nivolumab (Opdivo $\left.{ }^{\circledR}\right)$ as second line, regardless of PD-L1 expression, in patients with NSCLC progressed during or after platinum-based chemotherapy according to results of CheckMate 017 (squamous) and CheckMate 057 (non-squamous) [1, $2]$. In the same year, pembrolizumab (Keytruda $\left.{ }^{\circledR}\right)$ was approved by FDA as second line in squamous and non-squamous patients with PD-L1 expression, according to results of a large multicenter, open-label, multi-part study (keynote 010) [3]. But what news in 2016? The main results concerned immunocheck-point inhibitors. In October 24, FDA approved pembrolizumab (Keytruda ${ }^{\circledR}$ ) for the treatment of patients with metastatic NSCLC whose tumors express PD-L1 $\geq 50 \%$ as determined by an FDA approved test [4]. This is the first approval of a check-point inhibitor for the first line of lung cancer. Approval was based on results of two randomized, con-

Manuscript accepted for publication April 04, 2017

Operative Oncology Unit, Azienda Ospedaliera "Ospedali Riuniti Marche Nord", Presidio "San Salvatore" di Pesaro (Italy), via Lombroso, Pesaro 61121, Italy. Email: d.rossi63@libero.it

doi: https://doi.org/10.14740/wjon1017w trolled trials that demonstrated statistically significant improvements in progression-free survival (PFS) and overall survival (OS) for patients randomized to pembrolizumab compared with chemotherapy. In the first trial of 305 patients (keynote 024) who had no prior treatment for metastatic NSCLC and PD-L1 expression greater than or equal to $50 \%$, those who received pembrolizumab (200 mg every 3 weeks) had a significant improvement in PFS (HR: 0.50; 95\% CI: 0.37 - 0.68; $\mathrm{P}<0.001$ ) with a median PFS of 10.3 months versus 6.0 months for those receiving platinum-based chemotherapy. A pre-specified interim analysis demonstrated a statistically significant improvement in OS for patients randomized to pembrolizumab as compared with chemotherapy (HR: $0.60 ; 95 \%$ CI: $0.41-0.89 ; \mathrm{P}<0.005)$. In the second three-arm trial (keynote 010 , previously cited), 1,033 patients who were previously treated for metastatic NSCLC with PD-L1 expression greater than or equal to $1 \%$, randomized to pembrolizumab 2 $\mathrm{mg} / \mathrm{kg}$ every 3 weeks (HR: $0.71 ; 95 \% \mathrm{CI}: 0.58$ - 0.88; P < 0.001 ) or pembrolizumab $10 \mathrm{mg} / \mathrm{kg}$ every 3 weeks (HR: 0.61 ; 95\% CI: 0.49 - 0.75; P $<0.001$ ), had an improved OS compared with patients receiving docetaxel [3]. The median survival was 10.4 months in the pembrolizumab $2 \mathrm{mg} / \mathrm{kg}$ arm, 12.7 months in the pembrolizumab $10 \mathrm{mg} / \mathrm{kg}$ arm, and 8.5 months in the docetaxel arm. The most common side effects of treatment with pembrolizumab included decreased appetite, fatigue, nausea, dyspnea, cough, and constipation. Rare but serious adverse events included immune-mediated pneumonitis, colitis, hepatitis, endocrinopathies, and nephritis. Unlike pembrolizumab, nivolumab (Opdivo ${ }^{\circledR}$ ) fails to demonstrate a benefit in PFS and OS in the same setting of patients. In Checkmate 026 trial [5], 541 patients with $\mathrm{PD}-\mathrm{L} 1$ expression $\geq 5 \%$ were randomized to receive nivolumab $3 \mathrm{mg} / \mathrm{kg}$ every 2 weeks versus best chemotherapy. PFS was the main endpoint but was not met (4.2 months with nivolumab versus 5.9 months with chemotherapy; HR: $1.15 ; \mathrm{P}=0.25)$; OS was similar in the two arms of treatment (14.4 and 13.2 months for nivolumab and chemotherapy, respectively; HR: 1.02). Several explanations were proposed to clarify so different results in the same setting of patients. The main issue appears to be patient selection, whereas pembrolizumab trial was conducted in patients whose tumors showed at least 50\% PD-L1 expression, and nivolumab trial enrolled patients with PD-L1 expression $\geq 5 \%$. However, the same results were documented in a small subgroup of patients who had at least 50\% PD-L1 expression (HR for PFS: 1.1; HR for OS: 0.90); nevertheless, the trial was not statistically powered for this analysis. Other explanations may be that OS was better than historical data in the chemotherapy arm 
possibly due to a different proportion of women and Asian patients; moreover, there was a high crossover (60\%) among patients who progressed while receiving chemotherapy. This difference in efficacy between nivolumab and pembrolizumab could be elucidated by the ongoing phase III study CheckMate-227. This trial explores the potential of the combination of nivolumab plus ipilimumab for PD-L1 patients and nivolumab plus ipilimumab or nivolumab plus chemotherapy in PD-L1-negative patients. In 2016, ASCO meeting presented the results of CheckMate 012 [6], a phase $1 \mathrm{~b}$ study with the combination of nivolumab and ipilimumab in PD-L1 positive patients $(\geq 1 \%)$. Overall response rate (ORR) was $57 \%$ but in those patients with $\geq 50 \%$ PD-L1 expression, the ORR was $92 \%$; in patients who tested PD-L1 negative, the ORR with the combination regimen was $15 \%$. These data seem to go in a single direction: PD-L1 expression is crucial to maximize activity and efficacy of check-point inhibitors in order to administer "right drug to right patient" with a real improvement of cost-effectiveness. Concerning patients with non-squamous cell lung cancer and genetic mutations, we want to focus on data presented at ESMO meeting for afatinib, at ELCC for osimertinib and at ASCO meeting for alectinib. At ESMO meeting, updated results of LUX-LUNG 7 were presented [7]. In this trial, PFS (median 11.0 months with afatinib vs. 10.9 months with gefitinib; HR) and time-to-treatment failure (median 13.7 months with afatinib vs. 11.5 months with gefitinib) were significantly longer with afatinib than with gefitinib. Updated results show that median survival of patients treated with afatinib was 27.9 months compared to 24.5 months for those receiving gefitinib, without reaching significance. However, updated results confirmed the primary analysis concerning two of its three co-primary endpoints: PFS by independent review and time-to-treatment failure. We think that afatinib remains one of the three options in first-line treatment with gefitinib and erlotinib: a careful evaluation of different toxicity between drugs (skin rash and diarrhea for afatinib and erlotinib, AST/ ALT increase for gefitinib) should be made for the best tailored treatment ("right drug to right patient"). At European Lung Cancer Conference in Geneva, AURA expansion study with osimertinib was presented [8] and mature data were recently published by Tony Mok [9]. Osimertinib is a third generation EGFR tyrosine kinase inhibitor (TKI), highly effective in patients with T-790M mutation. The study included 60 patients from two phase I expansion cohorts of the AURA trial that had locally advanced or metastatic EGFR mutated NSCLC. Thirty patients received $80 \mathrm{mg}$ a day and 30 received $160 \mathrm{mg}$ a day in the first-line setting. The median follow-up was 16.6 months. The ORR was 77\%. Median PFS was 19.3 months for the 160 $\mathrm{mg}$ dose and has not yet been reached for the $80 \mathrm{mg}$ dose. Median duration of response has not been reached. The drug was well tolerated with few adverse events, particularly at the approved $80 \mathrm{mg}$ dose, where just $10 \%$ of patients required dose reduction to manage toxicities. At ASCO meeting in Chicago, preliminary results of J-ALEX study were presented [10]. This is a Japanese phase III trial that randomized patients to alectinib or crizotinib as first-line treatment in patients with ALKtranslocation. On December 11, 2015, FDA approved alectinib (Alecensa ${ }^{\circledR}$ ) for patients with ALK-rearrangement positive NSCLC that is refractory to crizotinib. The J-ALEX study, af- ter a planned interim analysis, was stopped for efficacy, as an improvement in PFS was already demonstrated. In the alectinib patients, the median PFS was not reached, compared with 10.2 months in the crizotinib patients, yielding an HR of 0.34 $(\mathrm{P}<0.0001)$. Toxicity was very low (constipation was the main side effects); grade 3 and 4 adverse events also occurred more frequently with crizotinib $(51.9 \%)$ than with alectinib $(26.2 \%)$. Crizotinib still remains the best option in patients with ROS-1 rearrangements: on March 11, 2016 FDA approved crizotinib (Xalkori ${ }^{\circledR}$ ) to treat people with advanced NSCLC whose tumors have a ROS-1 gene alteration [11]. The safety and efficacy of Xalkori for the treatment of patients with ROS-1 positive tumors were evaluated in a multi-center, single arm study of 50 patients with ROS-1 positive NSCLC. Results showed $66 \%$ of participants experienced a complete or partial shrinkage of their NSCLC tumors, an effect that lasted a median of 18.3 months.

In conclusion, 2016 was a very exciting year for the treatment of lung cancer with good news for non-squamous, regardless of genetic mutations or not.

\section{References}

1. Brahmer J, Reckamp KL, Baas P, Crino L, Eberhardt WE, Poddubskaya E, Antonia S, et al. Nivolumab versus Docetaxel in Advanced Squamous-Cell Non-Small-Cell Lung Cancer. N Engl J Med. 2015;373(2):123-135.

2. Borghaei H, Paz-Ares L, Horn L, Spigel DR, Steins M, Ready NE, Chow LQ, et al. Nivolumab versus Docetaxel in Advanced Nonsquamous Non-Small-Cell Lung Cancer. N Engl J Med. 2015;373(17):1627-1639.

3. Herbst RS, Baas P, Kim DW, Felip E, Perez-Gracia JL, Han JY, Molina J, et al. Pembrolizumab versus docetaxel for previously treated, PD-L1-positive, advanced nonsmall-cell lung cancer (KEYNOTE-010): a randomised controlled trial. Lancet. 2016;387(10027):1540-1550.

4. Reck M, Rodriguez-Abreu D, Robinson AG, Hui R, Csoszi T, Fulop A, Gottfried M, et al. Pembrolizumab versus Chemotherapy for PD-L1-Positive Non-Small-Cell Lung Cancer. N Engl J Med. 2016;375(19):1823-1833.

5. Socinski M, Creelan B, Horn L, et al. CheckMate 026: A phase 3 trial of nivolumab vs investigator's choice (IC) of platinum-based doublet chemotherapy (PT-DC) as first-line therapy for stage iv/recurrent programmed death ligand 1 (PD-L1) positive NSCLC. Ann Oncol. 2016;27(suppl 6):LBA7.

6. Hellmann MD, Gettinger SN, Goldman JW, et al. Checkmate 012: Safety and efficacy of first line nivolumab and ipilimumab in advanced NCSCLC. J Clin Oncol. 2016;34(abstr 3001).

7. Paz-Ares L, Tan EH, Zhang L, et al. Afatinib (A) vs gefitinib $(\mathrm{G})$ in patients (pts) with EGFR mutation-positive $($ EGFRm+) non-small-cell lung cancer (NSCLC): overall survival (OS) data. Ann Oncol. 2016;27(6)(abstr LBA43).

8. Ramalingam S. Osimertinib as first line treatment for EGFR mutation-positive advanced NSCLC: update efficacy and safety results from two phase I expansion cohorts. European lung Cancer Conference 2016; abstr 
LBA1 PR.

9. Mok TS, Wu YL, Ahn MJ, Garassino MC, Kim HR, Ramalingam SS, Shepherd FA, et al. Osimertinib or Platinum-Pemetrexed in EGFR T790M-Positive Lung Cancer. N Engl J Med. 2017;376(7):629-640.

10. Nokihara H, Hida T, Kondo M, et al. Alectinib (ALC) versus crizotinib (CRZ) in ALK-inhibitor naive ALK- positive non-small cell lung cancer (ALK+ NSCLC): Primary results from the J-ALEX study. Proc Am Soc Clin Oncol. 2016; Abstract 9008.

11. Shaw AT, Ou SH, Bang YJ, Camidge DR, Solomon BJ, Salgia R, Riely GJ, et al. Crizotinib in ROS1-rearranged non-small-cell lung cancer. $N$ Engl J Med. 2014;371(21):1963-1971. 